SUPPORTING INFORMATION

for

\title{
Two-Dimensional Silicon Fingerprints Reveal Dramatic Variations in the Sources of Particulate Matter in Beijing during 2013-2017
}

\author{
Xuezhi Yang ${ }^{1,2}$, Dawei Lu ${ }^{1}$, Jihua Tan ${ }^{2}$, Xu Sun ${ }^{1}$, Qinghua Zhang ${ }^{1}$, Luyao Zhang ${ }^{1}$, Yong Li ${ }^{1}$, \\ Weichao Wang ${ }^{1}$, Qian Liu ${ }^{1,2,3}$, Guibin Jiang ${ }^{1,2}$ \\ ${ }^{1}$ State Key Laboratory of Environmental Chemistry and Ecotoxicology, Research Center for Eco- \\ Environmental Sciences, Chinese Academy of Sciences, Beijing 100085, China \\ ${ }^{2}$ College of Resources and Environment, University of Chinese Academy of Sciences, Beijing \\ 100190, China \\ ${ }^{3}$ Institute of Environment and Health, Jianghan University, Wuhan 430056, China \\ Email: qianliu@ rcees.ac.cn
}

\section{Content}

1. Supporting Experimental Section

2. Supporting Tables

Table S1-S6.

3. Supporting Figures

Figs. S1-S10.

4. References for SI 


\section{Supporting Experimental Section}

\section{Sampling of Primary Source Samples for $\mathbf{P M}_{2.5}$}

Here we selected seven primary sources for $\mathrm{PM}_{2.5}$, i.e., coal burning, industrial emission, biomass burning, urban fugitive dust, soil dust, construction dust, and vehicle emission, which have been widely thought as major primary sources of $\mathrm{PM}_{2.5}$ in Beijing in the previous literature. ${ }^{1-5}$ The sampling procedures and characterization of the primary sources are the same as in our previous reports. ${ }^{5,6}$ The sampling sites for all primary source samples were around the Beijing city and shown in Figure $\mathbf{S 1 .}$ The soil dusts were collected at different directions and different depths around the Beijing city. The industrial emission samples were collected by air sleeves or electrostatic filters placed in the chimneys in steel and power plants in Tangshan, a neighbor city to Beijing. The coal burning samples were fly ashes from burning of honeycomb briquette and lump coals that are the most commonly used type of coals in the Beijing region. The fly ashes were collected onto polypropylene membrane filters with a dilution system and an impactor at a flow rate of $36 \mathrm{~L} / \mathrm{min}^{7}$ The vehicle emission samples were collected, following a previously reported method, ${ }^{5,8}$ by a high-volume air sampler (Echo Hi-Vol, Italy) in the middle section of Tanyugou Tunnel (3455 m in length; Changping District, Beijing) that only permits motor vehicles to pass through. The biomass burning samples were collected by burning straws from Daxing and Tongzhou Districts, Beijing. The urban fugitive dusts were collected at an altitude of $20 \mathrm{~m}$ by an SYC-3 auto-sampler (Laoshan, China). The construction dusts were collected at several construction sites in Beijing.

\section{Measurement of Water-Soluble Ions (WSI) Concentration}

The concentrations of WSI were measured according to the reported method. ${ }^{9}$ In short, each $\mathrm{PM}_{2.5}$ sample was extracted by ultrasonication with $10 \mathrm{~mL}$ of ultrapure water for half an hour. The solution was then filtered through a micro-porous membrane syringe filter (PTFE, pore size: $0.45 \mu \mathrm{m}$ ) before analysis. Two typical anions $\left(\mathrm{SO}_{4}{ }^{2-}\right.$ and $\left.\mathrm{NO}_{3}{ }^{-}\right)$were analyzed by ion chromatography (IC, Dionex ICS1000, USA) using an anion column (IC SI-52 4E, $4 \mathrm{~mm} \mathrm{ID} \times 250 \mathrm{~mm}$ ) with $4.5 \mathrm{mM} \mathrm{Na} 2 \mathrm{CO}_{3} / 0.8 \mathrm{mM}$ $\mathrm{NaHCO}_{3}$ as an eluent at a flow rate of $0.8 \mathrm{~mL} \mathrm{~min}^{-1}$. Blank samples were also analyzed to subtract the background signals from the samples $(n=3)$.

\section{Uncertainty Analysis for the "IsoSources" Model}

The "IsoSources" isotopic mixing model, a mathematical model recommended by the U.S. 
Environmental Protection Agency (U.S. EPA), has been widely used to estimate contributions of different endmembers based on isotopic data. ${ }^{10,11}$ Apart from the measurement errors, the uncertainties of the "IsoSources" model may raise from the following aspects: First, the assumption about the uniform distribution of the $\mathrm{Si}$ isotopic composition for each emission source may introduce some uncertainties. Second, the calculation does not involve the data in adjacent cities and regions, which may cause a systematic bias taking into account the interregional transport of $\mathrm{PM}_{2.5}$. In this study, the uncertainties from the isotopic mixing model are estimated in Table S6. It should be noted from Figure 2a that several primary sources (i.e., construction dust, soil dust, urban fugitive dust, and biomass burning) show similar Si isotopic fingerprints, suggesting that the $\delta^{30} \mathrm{Si}$-based method may not well resolve these sources and would introduce large uncertainties to the modeling results. Despite that, regarding coal burning, industrial emission, and vehicle emission that have received prior regulation during the APPCAP period, they show quite different Si isotopic fingerprints, which are favorable for using Si isotopic fingerprints to study their variations. As shown in Table S6, the RSDs for construction dust, soil dust, urban fugitive dust, and biomass burning (77\%-90\%) were larger than those for coal burning, industrial emission, and vehicle emission (21\%-92\%).

On the other hand, the uncertainties obtained here, especially for coal burning, industrial emission, and vehicle emission, were comparable to that obtained in other studies using the "IsoSources" model ${ }^{11 \text {, }}$ 12 and using the traditional PMF and CMB source apportionment models. ${ }^{13-15}$ Noteworthily, unlike the present method that uses only one tracer $\left(\delta^{30} \mathrm{Si}\right)$, the traditional methods require multiple chemical species as tracers (e.g., EC, $\mathrm{OC}, \mathrm{NO}_{3}{ }^{-}, \mathrm{SO}_{4}{ }^{2-}, \mathrm{NH}_{4}{ }^{+}$, and metal elements) in the calculation. The measurements of multiple chemical species may also bring in large uncertainties to the result. For example, the RSDs in the EC measurement could reach $37 \%-46 \%,{ }^{16}$ and the measurement of multiple metal concentrations might also cause significant uncertainties. ${ }^{2,17}$

\section{Validation of the Modelling Results}

The "IsoSources" model was used to quantitatively show the variation trends in primary sources based on the Si isotopic data. This model has been widely used and well validated in previous studies to estimate contributions of different endmembers based on isotopic data. ${ }^{10-12}$ Here we further validate the modelling results from the following aspects: (1) We have measured some traditional tracers (e.g., $\mathrm{SO}_{2}, \mathrm{NO}_{x}, \mathrm{SO}_{4}{ }^{2-}, \mathrm{NO}_{3}{ }^{-}$; see Figure S8), and the results well supported the modelling results. As shown in Figure $\mathbf{S 8 b}, \mathrm{SO}_{2}$ that is mainly emitted from coal burning and industrial emission showed a sharp 
decline during 2013-2015 and then changed slowly during 2015-2017. This trend was consistent with the variation trends of coal burning and industrial emission in Figure 2d. The reduction extent of $\mathrm{NO}_{x}$ was much smaller than that of $\mathrm{SO}_{2}$ from 2013 to in 2015 (Figure S8a), also corresponding to the increased contribution fraction of vehicle emission. (2) We have also compared our results with those obtained in the literature. The source apportionment result of 2013 was compared with a previous report using the traditional methods. ${ }^{1}$ Due to the different source classification and different sampling dates, it is difficult to directly compare the contribution percentages of different sources. Nevertheless, a similar order of primary source contributions were obtained (coal burning was the most, dusts were next, followed by biomass burning and traffic). Furthermore, the variation trends of various primary sources during 2013-2017 revealed by the "IsoSources" model were highly consistent with that estimated by bottom-up emission inventory model and chemical components of $\mathrm{PM}_{1},{ }^{18,19}$ that is, control of coal burning and industrial emission was found to contribute more to the $\mathrm{PM}_{2.5}$ reduction in Beijing than vehicle emission.

\section{Note for the SDs in $\delta^{29} \mathrm{Si}$ and $\delta^{30} \mathrm{Si}$ measured by MC-ICP-MS}

In the MC-ICP-MS measurement of Si isotope composition, we found that there was a high variability in the SDs for $\delta^{29} \mathrm{Si}$ and $\delta^{30} \mathrm{Si}$ (see Table S1). In some samples, the $\delta^{29} \mathrm{Si}$ and $\delta^{30} \mathrm{Si}$ did not change synchronously, i.e., a low $\delta^{29} \mathrm{Si}$ SD accompanying a high $\delta^{30} \mathrm{Si}$ SD. This phenomenon has also been observed in previous studies. ${ }^{5,20-24}$ We infer that this may be related to the special detection method of MC-ICP-MS. Because the three Si isotopes $\left({ }^{29} \mathrm{Si},{ }^{29} \mathrm{Si}\right.$, and $\left.{ }^{30} \mathrm{Si}\right)$ were detected by separate collectors $\left(\mathrm{L}^{4}{ }^{28} \mathrm{Si}, \mathrm{H} 1-{ }^{29} \mathrm{Si}, \mathrm{H} 6-{ }^{30} \mathrm{Si}\right.$; see Table S3), the signal fluctuation during the amplification and detection processes in the instrument might be independent, which therefore caused an inconsistent variations in the SD of $\delta^{29} \mathrm{Si}$ and $\delta^{30} \mathrm{Si}$.

Furthermore, it is worth noting that the SDs are independent of the values of $\delta^{30} \mathrm{Si}$ and $\delta^{29} \mathrm{Si}$ which are expressed as relative values to the standard material NIST SRM-8546 as zero value (Eq. 1-2). Therefore, the RSDs in 2015 were larger than those in other years, because the $\delta^{30} \mathrm{Si}$ values in 2015 were distributed closer to zero than in other years, rather than larger uncertainties for samples in 2015. 
110 Table S1. Meteorological conditions (relative humidity (RH), wind speed (WS) and temperature $(T)$ ), secondary aerosol precursors $\left(\mathbf{N O}_{\mathrm{x}}\right.$

111 and $\left.\mathrm{SO}_{2}\right)$, secondary species $\left(\mathrm{NO}_{3}{ }^{-}\right.$and $\left.\mathrm{SO}_{4}{ }^{2-}\right), \mathrm{PM}_{2.5}$ concentration, and the $\mathrm{Si}$ concentration $(\mathrm{Si})$, $\mathrm{Si}$ abundance $(\mathrm{Si} \%)$, and $\mathrm{Si}$ isotopic

112 composition $\left(\delta^{29} \mathrm{Si}\right.$ and $\left.\delta^{30} \mathrm{Si}\right)$ for all $\mathrm{PM}_{2.5}$ Samples (2013-2017) used in this study. ${ }^{\mathrm{a}}$

\begin{tabular}{|c|c|c|c|c|c|c|c|c|c|c|c|c|c|c|}
\hline Date & $\begin{array}{l}\text { RH } \\
(\%)\end{array}$ & $\begin{array}{c}\text { WS } \\
(\mathrm{m} / \mathrm{s})\end{array}$ & $\begin{array}{c}T \\
\left({ }^{\circ} \mathrm{C}\right) \\
\end{array}$ & $\begin{array}{c}\mathrm{NO}_{x} \\
(\mathrm{~nL} / \mathrm{L})\end{array}$ & $\begin{array}{c}\mathrm{SO}_{2} \\
(\mathrm{~nL} / \mathrm{L})\end{array}$ & $\begin{array}{c}\mathrm{NO}_{3}^{-} \\
\left(\mu \mathrm{g} / \mathrm{m}^{3}\right) \\
\end{array}$ & $\begin{array}{c}\mathrm{SO}_{4}{ }^{2-} \\
\left(\mu \mathrm{g} / \mathrm{m}^{3}\right)\end{array}$ & $\begin{array}{c}\mathrm{PM}_{2.5} \\
\left(\mu \mathrm{g} / \mathrm{m}^{3}\right)\end{array}$ & $\begin{array}{c}\mathrm{Si} \\
\left(\mu \mathrm{g} / \mathrm{m}^{3}\right) \\
\end{array}$ & $\begin{array}{l}\mathrm{Si} \% \\
(\%) \\
\end{array}$ & $\begin{array}{c}\delta^{29} \mathrm{Si} \\
(\%)\end{array}$ & $\begin{array}{l}\mathrm{SD}^{\mathrm{b}} \\
(\%) \\
\end{array}$ & $\begin{array}{c}\delta^{30} \mathrm{Si} \\
(\% 0)\end{array}$ & $\begin{array}{l}S^{b} \\
(\%)\end{array}$ \\
\hline 2013.01 .06 & 30.88 & 1.03 & -4.44 & 85.4 & 40.2 & 16.4 & 22.2 & 131 & 0.90 & 0.69 & -0.57 & 0.04 & -0.94 & 0.07 \\
\hline 2013.01 .11 & 55.84 & 1.24 & -2.66 & 134 & 47.8 & 26.1 & 52.0 & 241 & 2.09 & 0.87 & -0.76 & 0.09 & -1.43 & 0.17 \\
\hline 2013.01.18 & 43.33 & 0.77 & -1.98 & 155 & 30.4 & 31.0 & 39.3 & 211 & 0.64 & 0.30 & -0.38 & 0.11 & -1.04 & 0.12 \\
\hline 2013.01 .25 & 32.46 & 1.22 & -4.21 & 66.2 & 20.4 & 12.1 & 11.3 & 83.4 & 1.03 & 1.24 & -0.48 & 0.07 & -0.85 & 0.19 \\
\hline 2013.01 .29 & 80.77 & 0.91 & -2.26 & 180 & 43.0 & 69.3 & 112 & 307 & 1.30 & 0.42 & -0.69 & 0.12 & -1.21 & 0.16 \\
\hline 2013.02 .02 & 32.20 & 1.40 & -1.15 & 43.4 & 14.9 & 15.8 & 13.8 & 62.9 & 0.42 & 0.68 & -0.24 & 0.16 & -0.92 & 0.09 \\
\hline 2013.02 .08 & 29.59 & 1.59 & -6.88 & 25.6 & 20.7 & 7.56 & 30.7 & 111 & 0.70 & 0.63 & -0.35 & 0.05 & -1.04 & 0.14 \\
\hline 2013.02 .11 & 34.43 & 1.42 & -3.68 & 26.1 & 21.5 & 6.79 & 31.5 & 85.4 & 5.61 & 6.58 & -0.41 & 0.06 & -0.70 & 0.11 \\
\hline 2013.02 .23 & 23.99 & 0.81 & 4.02 & 108 & 59.5 & 16.8 & 43.1 & 113 & 0.99 & 0.87 & -0.73 & 0.16 & -1.42 & 0.09 \\
\hline 2013.02 .24 & 33.23 & 0.85 & 3.11 & 81.2 & 29.7 & 28.2 & 34.3 & 235 & 1.68 & 0.72 & -0.75 & 0.04 & -1.56 & 0.13 \\
\hline 2013.02 .27 & 40.80 & 1.59 & 7.42 & 92.9 & 24.3 & 30.8 & 53.6 & 213 & 1.48 & 0.70 & -0.98 & 0.06 & -1.93 & 0.09 \\
\hline 2013.12.01 & 26.93 & 0.77 & 5.94 & 38.5 & 15.6 & 6.41 & 24.4 & 104 & 1.23 & 1.18 & -0.54 & 0.07 & -1.11 & 0.09 \\
\hline 2013.12.06 & 33.93 & 0.82 & 4.98 & 131 & 40.6 & 21.2 & 12.4 & 159 & 1.58 & 0.99 & -0.73 & 0.09 & -1.43 & 0.14 \\
\hline 2013.12.07 & 54.13 & 0.58 & 3.45 & 92.7 & 27.5 & 35.8 & 39.4 & 296 & 3.35 & 1.13 & -0.89 & 0.07 & -1.75 & 0.13 \\
\hline 2013.12 .22 & 33.44 & 0.75 & -1.53 & 82.7 & 34.4 & 26.2 & 19.7 & 149 & 0.94 & 0.63 & -0.69 & 0.06 & -1.22 & 0.11 \\
\hline 2015.11 .29 & 59.50 & 0.16 & 1.89 & 122 & 12.4 & 33.0 & 26.8 & 227 & 2.12 & 0.93 & -0.16 & 0.05 & -0.31 & 0.24 \\
\hline 2015.11 .30 & 77.75 & 0.00 & 0.52 & 172 & 10.3 & 78.8 & 104 & 558 & 2.29 & 0.41 & 0.02 & 0.01 & 0.19 & 0.04 \\
\hline 2015.12 .01 & 56.71 & 0.82 & 1.81 & 90.8 & 9.32 & 50.6 & 40.7 & 336 & 2.81 & 0.84 & -0.04 & 0.08 & 0.09 & 0.12 \\
\hline 2015.12 .02 & 24.83 & 3.38 & 0.90 & 9.42 & 3.31 & 0.25 & 0.70 & 66.9 & 4.91 & 7.34 & -0.30 & 0.01 & -0.33 & 0.06 \\
\hline 2015.12 .03 & 27.01 & 3.06 & -0.06 & 15.3 & 4.55 & 0.09 & 1.13 & 45.9 & 3.36 & 7.33 & -0.12 & 0.04 & -0.02 & 0.15 \\
\hline
\end{tabular}




\begin{tabular}{|c|c|c|c|c|c|c|c|c|c|c|c|c|c|c|}
\hline 2015.12.04 & 27.46 & 0.84 & 4.08 & 57.5 & 7.86 & 2.55 & 1.42 & 81.0 & 3.47 & 4.28 & -0.04 & 0.12 & -0.13 & 0.22 \\
\hline 2015.12.05 & 38.84 & 0.25 & 3.89 & 77.0 & 9.45 & 5.00 & 2.40 & 111 & 2.92 & 2.63 & -0.01 & 0.10 & 0.22 & 0.03 \\
\hline 2015.12.06 & 55.55 & 0.05 & 2.77 & 104 & 15.4 & 20.3 & 12.0 & 192 & 1.01 & 0.53 & 0.00 & 0.07 & -0.03 & 0.16 \\
\hline 2015.12.07 & 69.25 & 0.05 & 2.37 & 136 & 13.3 & 25.0 & 23.1 & 266 & 0.67 & 0.25 & -0.02 & 0.01 & 0.23 & 0.21 \\
\hline 2015.12.08 & 81.91 & 0.09 & 1.87 & 102 & 9.23 & 32.0 & 31.6 & 265 & 0.43 & 0.16 & 0.06 & 0.07 & -0.05 & 0.13 \\
\hline 2015.12.09 & 72.63 & 0.32 & 3.89 & 87.3 & 11.3 & 47.1 & 34.9 & 286 & 0.65 & 0.23 & -0.08 & 0.01 & -0.24 & 0.06 \\
\hline 2015.12 .10 & 30.96 & 1.20 & 4.55 & 46.3 & 8.05 & 1.60 & 1.45 & 51.4 & 0.92 & 1.80 & -0.07 & 0.03 & -0.24 & 0.02 \\
\hline 2015.12.12 & 51.39 & 0.04 & 2.09 & 126 & 12.5 & 15.3 & 6.89 & 172 & 1.58 & 0.92 & 0.08 & 0.05 & 0.05 & 0.15 \\
\hline 2015.12 .13 & 66.78 & 0.11 & 2.45 & 108 & 12.5 & 27.7 & 22.9 & 291 & 1.83 & 0.63 & -0.01 & 0.01 & 0.00 & 0.22 \\
\hline 2015.12 .14 & 27.79 & 2.74 & 2.93 & 13.6 & 4.56 & 4.13 & 4.95 & 51.1 & 0.79 & 1.56 & -0.12 & 0.02 & -0.29 & 0.18 \\
\hline 2015.12 .15 & 25.52 & 2.63 & -0.49 & 10.2 & 3.48 & 0.38 & 1.37 & 44.9 & 2.09 & 4.65 & -0.14 & 0.05 & -0.14 & 0.09 \\
\hline 2015.12 .16 & 28.06 & 1.53 & -1.96 & 32.1 & 5.11 & 0.81 & 1.38 & 47.2 & 1.57 & 3.32 & -0.13 & 0.04 & -0.26 & 0.10 \\
\hline 2015.12 .17 & 31.27 & 0.36 & 1.34 & 115 & 12.5 & 0.48 & 1.17 & 167 & 1.71 & 1.03 & 0.02 & 0.08 & 0.03 & 0.13 \\
\hline 2015.12.18 & 39.17 & 0.38 & 2.37 & 123 & 11.6 & 0.93 & 0.85 & 166 & 0.57 & 0.34 & -0.05 & 0.04 & -0.10 & 0.12 \\
\hline 2015.12 .20 & 64.98 & 0.01 & -0.05 & 113 & 12.5 & 7.61 & 4.23 & 168 & 1.10 & 0.66 & -0.10 & 0.02 & -0.16 & 0.16 \\
\hline 2015.12.21 & 60.73 & 0.05 & 1.42 & 141 & 13.7 & 18.5 & 9.95 & 308 & 1.49 & 0.48 & -0.02 & 0.07 & -0.02 & 0.17 \\
\hline 2015.12.22 & 78.13 & 0.25 & -0.89 & 142 & 11.8 & 56.0 & 60.3 & 400 & 1.07 & 0.27 & 0.09 & 0.05 & -0.01 & 0.08 \\
\hline 2015.12 .23 & 36.61 & 0.25 & 3.46 & 85.4 & 14.5 & 15.6 & 12.2 & 150 & 1.75 & 1.17 & 0.07 & 0.06 & 0.03 & 0.14 \\
\hline 2015.12.24 & 49.26 & 0.97 & 1.43 & 104 & 8.12 & 28.0 & 28.9 & 265 & 2.81 & 1.06 & 0.05 & 0.05 & -0.07 & 0.14 \\
\hline 2015.12 .25 & 82.13 & 0.11 & -2.29 & 163 & 6.06 & 73.0 & 74.4 & 575 & 1.15 & 0.20 & -0.12 & 0.03 & -0.19 & 0.06 \\
\hline 2015.12.26 & 67.24 & 0.51 & -3.04 & 44.8 & 5.81 & 16.2 & 20.6 & 144 & 1.34 & 0.93 & -0.16 & 0.07 & -0.27 & 0.07 \\
\hline 2015.12.27 & 54.19 & 0.11 & -5.74 & 106 & 9.26 & 5.73 & 6.20 & 117 & 1.45 & 1.23 & 0.05 & 0.07 & -0.02 & 0.09 \\
\hline 2015.12.28 & 51.13 & 0.01 & -0.44 & 145 & 13.7 & 21.1 & 16.1 & 215 & 1.38 & 0.64 & 0.05 & 0.08 & 0.22 & 0.15 \\
\hline 2015.12.29 & 50.59 & 0.33 & 1.32 & 136 & 15.4 & 28.1 & 25.9 & 290 & 0.79 & 0.27 & -0.06 & 0.06 & -0.11 & 0.04 \\
\hline 2015.12.30 & 31.59 & 1.34 & 2.15 & 27.5 & 6.61 & 0.84 & 1.32 & 58.5 & 0.21 & 0.36 & -0.10 & 0.04 & -0.17 & 0.03 \\
\hline 2016.11.31 & 43.44 & 0.00 & 0.58 & 56.3 & 14.3 & 10.4 & 4.98 & 155 & 1.60 & 1.03 & -0.03 & 0.04 & -0.16 & 0.03 \\
\hline 2016.12.01 & 30.60 & 0.27 & 5.11 & 71.0 & 8.27 & 6.67 & 3.49 & 55.3 & 1.22 & 2.20 & 0.03 & 0.03 & 0.07 & 0.14 \\
\hline 2016.12.02 & 38.36 & 0.01 & 5.18 & 133 & 10.6 & 19.9 & 7.03 & 112 & 1.36 & 1.21 & -0.14 & 0.01 & -0.25 & 0.01 \\
\hline 2016.12 .03 & 56.19 & 0.01 & 4.41 & 185 & 9.41 & 56.9 & 35.9 & 302 & 2.27 & 0.75 & -0.16 & 0.01 & -0.36 & 0.06 \\
\hline
\end{tabular}




\begin{tabular}{|c|c|c|c|c|c|c|c|c|c|c|c|c|c|c|}
\hline 2016.12.04 & 45.67 & 0.24 & 5.84 & 58.7 & 8.44 & 32.1 & 18.1 & 133 & 2.50 & 1.88 & -0.18 & 0.02 & -0.24 & 0.07 \\
\hline 2016.12 .05 & 19.52 & 1.51 & 2.18 & 55.1 & 6.11 & 1.97 & 0.95 & 32.6 & 1.80 & 5.52 & 0.04 & 0.03 & -0.00 & 0.00 \\
\hline 2016.12.06 & 31.42 & 0.05 & 2.92 & 210 & 15.0 & 16.3 & 4.76 & 124 & 2.59 & 2.10 & -0.08 & 0.05 & -0.13 & 0.01 \\
\hline 2016.12.07 & 45.40 & 0.32 & 5.02 & 129 & 10.6 & 27.1 & 16.2 & 161 & 1.79 & 1.11 & -0.07 & 0.02 & -0.12 & 0.09 \\
\hline 2016.12.08 & 23.80 & 2.41 & 4.08 & 29.0 & 3.99 & 1.10 & 0.74 & 15.9 & 1.31 & 8.21 & 0.08 & 0.08 & 0.08 & 0.01 \\
\hline 2016.12.09 & 33.21 & 0.12 & 1.12 & 61.2 & 6.85 & 4.88 & 1.92 & 39.4 & 1.12 & 2.85 & 0.03 & 0.06 & 0.06 & 0.13 \\
\hline 2016.12 .10 & 34.93 & 0.00 & 1.53 & 127 & 13.2 & 8.47 & 3.65 & 57.1 & 1.75 & 3.08 & 0.03 & 0.07 & -0.00 & 0.02 \\
\hline 2016.12 .11 & 51.59 & 0.12 & 1.27 & 141 & 14.1 & 31.2 & 21.3 & 197 & 1.76 & 0.89 & -0.14 & 0.02 & -0.26 & 0.19 \\
\hline 2016.12.12 & 49.02 & 0.06 & 3.77 & 115 & 15.3 & 45.2 & 25.2 & 190 & 1.00 & 0.53 & -0.14 & 0.03 & -0.41 & 0.04 \\
\hline 2016.12 .13 & 24.34 & 0.19 & 0.66 & 38.0 & 5.23 & 2.27 & 1.00 & 33.4 & 0.56 & 1.69 & -0.24 & 0.10 & -0.41 & 0.01 \\
\hline 2016.12 .14 & 28.06 & 0.34 & -0.27 & 57.0 & 7.86 & 5.89 & 2.09 & 46.3 & 1.03 & 2.23 & 0.07 & 0.07 & 0.02 & 0.12 \\
\hline 2016.12 .15 & 23.88 & 1.40 & 1.38 & 61.0 & 6.19 & 1.38 & 1.08 & 29.6 & 1.17 & 3.96 & 0.04 & 0.03 & 0.22 & 0.01 \\
\hline 2016.12 .16 & 34.96 & 0.01 & 2.48 & 133 & 13.8 & 18.4 & 9.49 & 133 & 1.64 & 1.23 & -0.04 & 0.09 & -0.03 & 0.16 \\
\hline 2016.12 .17 & 47.12 & 0.00 & 2.16 & 128 & 16.1 & 41.3 & 25.5 & 204 & 1.23 & 0.60 & -0.17 & 0.05 & -0.43 & 0.11 \\
\hline 2016.12 .18 & 52.50 & 0.00 & 2.72 & 150 & 15.1 & 52.1 & 28.1 & 223 & 1.15 & 0.52 & -0.37 & 0.07 & -0.68 & 0.11 \\
\hline 2016.12.19 & 47.21 & 0.00 & 4.66 & 159 & 17.3 & 57.4 & 30.8 & 259 & 1.60 & 0.62 & -0.27 & 0.05 & -0.52 & 0.01 \\
\hline 2016.12 .20 & 68.93 & 0.01 & 1.42 & 162 & 4.19 & 56.5 & 44.6 & 291 & 1.01 & 0.35 & -0.14 & 0.03 & -0.41 & 0.05 \\
\hline 2016.12.21 & 54.39 & 0.42 & 3.44 & 131 & 6.13 & 60.4 & 45.3 & 276 & 1.01 & 0.36 & -0.11 & 0.03 & -0.35 & 0.12 \\
\hline 2016.12.22 & 28.67 & 1.92 & 0.73 & 22.2 & 2.42 & 0.64 & 2.39 & 9.9 & 0.98 & 9.96 & -0.33 & 0.09 & -0.64 & 0.03 \\
\hline 2017.12 .13 & 23.62 & 0.01 & -2.30 & 75.7 & 4.26 & 8.16 & 2.09 & 51.5 & 1.32 & 2.56 & -0.36 & 0.06 & -0.70 & 0.11 \\
\hline 2017.12 .14 & 31.90 & 1.82 & -1.41 & 52.7 & 2.94 & 10.2 & 3.53 & 60.9 & 1.44 & 2.37 & -0.04 & 0.06 & -0.23 & 0.03 \\
\hline 2017.12 .15 & 17.80 & 2.70 & -2.15 & 4.50 & 0.89 & 0.27 & 1.19 & 12.9 & 1.60 & 12.4 & -0.62 & 0.01 & -1.23 & 0.18 \\
\hline 2017.12.16 & 22.10 & 0.66 & -2.05 & 31.9 & 1.58 & 0.63 & 0.68 & 15.2 & 1.11 & 7.30 & -0.15 & 0.01 & -0.34 & 0.06 \\
\hline 2017.12 .17 & 15.96 & 1.02 & 3.95 & 21.0 & 2.60 & 1.74 & 0.95 & 23.5 & 1.49 & 6.33 & -0.34 & 0.06 & -0.58 & 0.06 \\
\hline 2017.12.18 & 19.94 & 1.17 & 0.81 & 21.9 & 1.66 & 0.38 & 0.53 & 21.2 & 1.14 & 5.39 & 0.01 & 0.12 & -0.12 & 0.01 \\
\hline 2017.12.19 & 22.18 & 0.85 & 3.34 & 39.3 & 3.83 & 4.17 & 1.45 & 35.6 & 1.53 & 4.30 & -0.33 & 0.03 & -0.70 & 0.08 \\
\hline 2017.12 .20 & 27.92 & 0.54 & 3.46 & 65.3 & 2.33 & 1.92 & 0.93 & 24.8 & 0.35 & 1.43 & 0.87 & 0.06 & 1.72 & 0.12 \\
\hline 2017.12 .21 & 27.85 & 0.62 & 4.00 & 103 & 4.49 & 7.29 & 2.15 & 55.8 & 1.51 & 2.71 & -0.15 & 0.05 & -0.31 & 0.01 \\
\hline 2017.12.22 & 19.23 & 0.44 & 4.12 & 30.9 & 3.53 & 0.92 & 0.55 & 21.1 & 0.98 & 4.62 & -0.05 & 0.03 & -0.27 & 0.12 \\
\hline
\end{tabular}




\begin{tabular}{|c|c|c|c|c|c|c|c|c|c|c|c|c|c|c|}
\hline 2017.12.23 & 23.04 & 0.75 & 3.99 & 45.1 & 4.90 & 3.70 & 2.08 & 30.3 & 1.65 & 5.46 & -0.32 & 0.03 & -0.72 & 0.03 \\
\hline 2017.12.24 & 18.32 & 0.67 & 0.09 & 21.2 & 2.62 & 2.10 & 0.85 & 14.4 & 0.91 & 6.31 & -0.07 & 0.01 & -0.23 & 0.02 \\
\hline 2017.12.27 & 56.80 & 0.25 & -1.41 & 69.7 & 1.39 & 16.4 & 13.6 & 81.7 & 1.05 & 1.28 & 0.00 & 0.09 & -0.20 & 0.06 \\
\hline 2017.12.28 & 63.66 & 0.07 & -0.89 & 82.3 & 1.60 & 39.6 & 18.2 & 130 & 0.91 & 0.70 & -0.12 & 0.01 & -0.32 & 0.10 \\
\hline 2017.12.29 & 71.96 & 0.08 & -0.57 & 130 & 1.68 & 48.6 & 34.9 & 186 & 0.76 & 0.41 & -0.19 & 0.03 & -0.40 & 0.01 \\
\hline 2017.12.30 & 17.48 & 0.98 & 1.99 & 30.0 & 1.32 & 0.54 & 1.12 & 22.8 & 2.16 & 9.49 & -0.37 & 0.03 & -0.74 & 0.08 \\
\hline
\end{tabular}

${ }^{\text {a }}$ The samples in 2013 were randomly collected on haze days. The sample information for 2013 were adopted from our previous paper. ${ }^{6}$ The Si isotopic composition measurement for 2013 and 2015-2017 was conducted on the same instrument and with the same QA/QC procedures to ensure no systematic bias between years.

${ }^{\mathrm{b}}$ It is worth noting that there is a high variability in the reported SD for $\delta^{29} \mathrm{Si}$ and $\delta^{30} \mathrm{Si}$. In some samples, the $\delta^{29} \mathrm{Si}$ and $\delta^{30} \mathrm{Si}$ did not change synchronously, i.e., a low $\delta^{29} \mathrm{Si} \mathrm{SD}$ accompanying a high $\delta^{30} \mathrm{Si} \mathrm{SD}$. This phenomenon has also been observed in previous studies. ${ }^{5,20-24}$ We infer that this may be related to the special detection method of MC-ICP-MS. Because the three Si isotopes $\left({ }^{29} \mathrm{Si},{ }^{29} \mathrm{Si}\right.$, and $\left.{ }^{30} \mathrm{Si}\right)$ were detected by separate collectors $\left(\mathrm{L} 4-{ }^{28} \mathrm{Si}, \mathrm{H} 1-{ }^{29} \mathrm{Si}, \mathrm{H} 6-{ }^{30} \mathrm{Si}\right.$; see Table S3), the signal fluctuation during the amplification and detection processes in the instrument might be independent, which therefore caused an inconsistent variations in the SD of $\delta^{29} \mathrm{Si}$ and $\delta^{30} \mathrm{Si}$. Furthermore, the SDs are independent of the values of $\delta^{30} \mathrm{Si}$ and $\delta^{29} \mathrm{Si}$ which are expressed as relative values to the standard material NIST SRM-8546 as zero value (Eq. 1-2). Therefore, the RSDs in 2015 were larger than those in other years, because the $\delta^{30} \mathrm{Si}$ values in 2015 were distributed closer to zero than in other years, rather than larger uncertainties for samples in 2015. 
Table S2. The concentrations of $\mathrm{Si}$ and $\mathrm{Na}$ in the filtrate after the cation-exchange column purification.

\begin{tabular}{lllllllll}
\hline & & & $\mathrm{PM}_{2.5-1}$ & $\mathrm{PM}_{2.5-2}$ & $\mathrm{PM}_{2.5-3}$ & $\mathrm{PM}_{2.5-4}$ & $\mathrm{PM}_{2.5-5}$ & $\mathrm{PM}_{2.5-6}$ \\
& SRM-8546 & IRMM-017 & (2016.12.08) & $(2016.12 .09)$ & $(2016.12 .10)$ & $(2016.12 .11)$ & $(2016.12 .12)$ & $(2016.12 .13)$ \\
\hline Si concentration (mg/L) & 0.65 & 0.65 & 0.61 & 0.67 & 0.65 & 0.60 & 0.60 & 0.66 \\
Na concentration (mg/L) & 0.005 & 0.03 & 0.07 & 0.07 & 0.05 & 0.06 & 0.09 & 0.09 \\
\hline
\end{tabular}

${ }^{a}$ The $\mathrm{Si}$ concentration in solutions loaded onto the column was $\sim 4 \mathrm{mg} / \mathrm{L}$. Prior to MC-ICP-MS measurement, the Si concentration in all samples were adjusted to the same level (difference $<10 \%$ ).

${ }^{\mathrm{b}}$ Results showed that after the cation-exchange column purification, the Na content in all tested samples was below $0.09 \mathrm{mg} / \mathrm{L}$. According to the results in Figure S2, this would only cause a deviation in $\delta^{30} \mathrm{Si}$ of less than $0.03 \%$. This value was much less than the method uncertainty $(0.10 \%)$, indicating that the matrix effect of $\mathrm{Na}$ in this study was negligible. 
131 Table S3. Parameters for Si isotopic composition measurement by MC-ICP-MS.

Instrument settings

RF power

Nebulizer pressure

Hot gas (Ar) flow rate

Membrane gas flow rate

Sample introduction

Sampler cone (nickel)

Skimmer cone (nickel)

Lens settings

Torch

Collector

Data acquisition parameters

Scan type

Measurement mode

Measurement intensity $\left({ }^{28} \mathrm{Si}\right)$

Blank signal $(\mathrm{pH} \sim 2) \mathrm{HCl}$ of ${ }^{28} \mathrm{Si}$

Magnet delay time

Number of blocks

Integration time
$1300 \mathrm{~W}$

$27.6 \mathrm{psi}$

$0.25 \mathrm{~L} / \mathrm{min}$

$2.68 \mathrm{~L} / \mathrm{min}$

DeSolvation Nebulizer (DSN-100)

PFA nebulizer, uptake rate $\sim 70 \mu \mathrm{L} / \mathrm{min}$

“experimental” WA cone (Nu Instruments)

“experimental” WA cone (Nu Instruments)

Optimized for maximum analytical signal intensity glass

L4- ${ }^{28} \mathrm{Si}, \mathrm{H} 1-{ }^{29} \mathrm{Si}, \mathrm{H} 6-{ }^{30} \mathrm{Si}$

Static measurements

medium-resolution with standard-sample-standard bracketing

3.6 to $7.5 \mathrm{~V}$

20 to $40 \mathrm{mV}$

$2 \mathrm{~s}$

3 block, 10 cycles

$10 \mathrm{~s}$ 
Table S4. Parameters used in the estimate of secondary aerosol contribution to PM$_{2.5}$ from 2013 to 2017 by the Si-dilution method. ${ }^{6}$

\begin{tabular}{|c|c|c|c|c|c|}
\hline & Coal combustion & Industrial emission & Biomass burning & Vehicle exhaust & Dust $^{\mathrm{a}}$ \\
\hline Contribution- $2013^{b}$ & 0.380 & 0.193 & 0.102 & 0.057 & 0.269 \\
\hline Contribution-2015 & 0.049 & 0.074 & 0.124 & 0.277 & 0.476 \\
\hline Contribution-2016 & 0.060 & 0.089 & 0.144 & 0.197 & 0.510 \\
\hline Contribution- $2017^{b}$ & 0.098 & 0.134 & 0.167 & 0.120 & 0.480 \\
\hline Si abundance $\left(C_{i}\right)^{\mathrm{c}}$ & $8.06 \%$ & $0.79 \%$ & $1.01 \%$ & $0.20 \%$ & $14.0 \%$ \\
\hline & $C_{\text {pri }}{ }^{\mathrm{d}}$ & $C_{\text {PM2.5 }}{ }^{\mathrm{e}}$ & & $f_{\mathrm{s}}^{\mathrm{f}}$ & \\
\hline 2013 & 0.0710 & 0.0118 & & $83.40 \%{ }^{\mathrm{g}}$ & \\
\hline 2015 & 0.0730 & 0.0155 & & $78.80 \%$ & \\
\hline 2016 & 0.0788 & 0.0230 & & $70.80 \%$ & \\
\hline 2017 & 0.0781 & 0.0457 & & $41.50 \%$ & \\
\hline
\end{tabular}

${ }^{a}$ In the calculation, soil, construction, and urban fugitive dust were considered as a single source (i.e., dust). ${ }^{6}$

${ }^{\mathrm{b}}$ The contributions of primary sources $\left(f_{i}\right)$ were calculated from the Si isotopic fingerprints by the "IsoSources" model (Table S6). In principle, the $f_{\mathrm{s}}$ for one year can be estimated in two ways: (1) calculate the $f_{\mathrm{s}}$ for each day, and then obtain the annual mean $f_{\mathrm{s}}$; (2) calculate the annual mean $C_{\text {pri }}$ and $C_{\mathrm{PM} 2.5}$, and then obtain the annual mean $f_{\mathrm{s}}$. In the present study, because the "IsoSources" model can only give the annually mean contribution fractions for primary sources rather than daily data, we used the latter way to obtain the $f_{\mathrm{s}}$.

${ }^{\mathrm{c}}$ The mean $\mathrm{Si}$ abundances of primary sources $\left(C_{i}\right)$ were adopted from the data in our previous articles. ${ }^{5,6}$

${ }^{\mathrm{d}} C_{\text {pri }}$ represents mean $\mathrm{Si}$ abundance in primary particles. It was calculated by the Eq: $C_{\text {pri }}=\sum\left(C_{i} \times f_{i}\right){ }^{6}$

${ }^{\mathrm{e}} \mathrm{CPM}_{\mathrm{PM} .5}$ represents mean $\mathrm{Si}$ abundance in $\mathrm{PM}_{2.5}$.

${ }^{\mathrm{g}}$ Note that, in the previous study, the contributions of primary sources $\left(f_{i}\right)$ were obtained from the MEIC database ${ }^{25}$ while in this study the $f_{i}$ values were estimated from the "IsoSources" model. The estimated secondary aerosol contributions in 2013 in Beijing from these two methods are actually very close $\left(79.2 \%\right.$ in the previous study ${ }^{6}$ and $83.4 \%$ in this study), suggesting that the results obtained here are reliable and reasonable. 
147 Table S5. Correlation coefficients $(R)$ for meteorological conditions (relative humidity (RH), 148 wind speed (WS), and temperature $(T)$ ), secondary aerosol precursors $\left(\mathrm{NO}_{\underline{x}}\right.$ and $\left.\mathrm{SO}_{2}\right)$, and 149 secondary species $\left(\mathrm{NO}_{3}{ }^{-}\right.$and $\left.\mathbf{S O}_{4}{ }^{2-}\right)$ with the $\mathbf{P M}_{2.5}$ concentration and $\mathrm{Si}$ isotopic composition 150 during 2013-2017.

\begin{tabular}{|c|c|c|c|c|c|c|c|c|}
\hline & $\mathrm{RH}$ & WS & $T$ & $\mathrm{NO}_{x}$ & $\mathrm{SO}_{2}$ & $\mathrm{NO}_{3}{ }^{-}$ & $\mathrm{SO}_{4}{ }^{2-}$ & $\mathrm{PM}_{2.5}$ \\
\hline $\mathrm{PM}_{2.5-2013}$ & $0.81 * * \mathrm{a}$ & -0.38 & 0.23 & $0.72 * *$ & 0.35 & 0.25 & $0.71 * *$ & 1.00 \\
\hline $\mathrm{PM}_{2.5-2015}$ & $0.82 * *$ & $-0.55^{* *}$ & -0.05 & $0.82 * *$ & 0.34 & $0.95 * *$ & $0.92 * *$ & 1.00 \\
\hline $\mathrm{PM}_{2.5}-2016$ & $0.93 * *$ & $-0.57 * *$ & 0.33 & $0.78 * *$ & $0.44 * \mathrm{~b}$ & $0.98 * *$ & $0.96 * *$ & 1.00 \\
\hline $\mathrm{PM}_{2.5-2017}$ & $0.95 * *$ & -0.47 & -0.30 & $0.84 * *$ & -0.16 & $0.99 * *$ & $0.96 * *$ & 1.00 \\
\hline $\mathrm{PM}_{2.5 \text {-all }}$ & $0.84 * *$ & $-0.44 * *$ & 0.00 & $0.74 * *$ & 0.29 & $0.87 * *$ & $0.81 * *$ & 1.00 \\
\hline$\delta^{30} \mathrm{Si}-2013$ & -0.25 & 0.22 & $-0.73 * *$ & -0.38 & -0.31 & -0.42 & -0.30 & $-0.66 * *$ \\
\hline$\delta^{30} \mathrm{Si}-2015$ & 0.24 & $-0.44^{*}$ & -0.19 & $0.52 * *$ & $0.45^{*}$ & 0.24 & 0.24 & 0.33 \\
\hline$\delta^{30} \mathrm{Si}-2016$ & -0.58 & 0.26 & 0.05 & -0.31 & -0.23 & $-0.64 * *$ & $-0.60 * *$ & $-0.57 * *$ \\
\hline$\delta^{30} \mathrm{Si}-2017$ & 0.12 & -0.31 & 0.24 & 0.23 & -0.05 & -0.01 & -0.02 & -0.01 \\
\hline$\delta^{30} \mathrm{Si}$-all & 0.11 & -0.23 & 0.08 & 0.03 & $-0.60 * *$ & -0.13 & $-0.31 * *$ & -0.03 \\
\hline
\end{tabular}
a**Significance at 0.001 level $(P<0.001),{ }^{b *}$ Significance at 0.05 level $(P<0.05)$. 
Table S6. The detailed source apportionment results of $\mathbf{P M}_{2.5}$ during 2013-2017 calculated by the model of "IsoSources".

\begin{tabular}{|c|c|c|c|c|c|c|c|c|c|c|c|c|c|c|}
\hline Date & $\begin{array}{c}\text { Coal } \\
\text { burning }\end{array}$ & SD & $\begin{array}{l}\text { Industrial } \\
\text { emission }\end{array}$ & SD & $\begin{array}{c}\text { Biomass } \\
\text { burning }\end{array}$ & $\mathrm{SD}$ & $\begin{array}{c}\text { Urban } \\
\text { fugitive } \\
\text { dust }\end{array}$ & SD & Soil dust & SD & $\begin{array}{c}\text { Construction } \\
\text { dust }\end{array}$ & SD & $\begin{array}{l}\text { Vehicle } \\
\text { emission }\end{array}$ & $\mathrm{SD}$ \\
\hline $2013-1^{a}$ & 0.380 & 0.098 & 0.193 & 0.166 & 0.102 & 0.090 & 0.098 & 0.087 & 0.093 & 0.083 & 0.078 & 0.070 & 0.057 & 0.052 \\
\hline $2015-1^{a}$ & 0.049 & 0.045 & 0.074 & 0.066 & 0.124 & 0.108 & 0.132 & 0.115 & 0.142 & 0.124 & 0.202 & 0.168 & 0.277 & 0.113 \\
\hline $2016-1^{a}$ & 0.060 & 0.052 & 0.089 & 0.076 & 0.144 & 0.125 & 0.152 & 0.132 & 0.163 & 0.142 & 0.195 & 0.152 & 0.197 & 0.110 \\
\hline $2017-1^{\mathrm{a}}$ & 0.098 & 0.067 & 0.134 & 0.101 & 0.167 & 0.145 & 0.166 & 0.144 & 0.164 & 0.141 & 0.150 & 0.123 & 0.120 & 0.093 \\
\hline $2013-2^{b}$ & 0.200 & 0.094 & 0.202 & 0.154 & 0.141 & 0.122 & 0.136 & 0.117 & 0.130 & 0.112 & 0.110 & 0.095 & 0.082 & 0.071 \\
\hline $2015-2^{b}$ & 0.058 & 0.051 & 0.086 & 0.075 & 0.142 & 0.123 & 0.150 & 0.130 & 0.160 & 0.139 & 0.197 & 0.157 & 0.207 & 0.111 \\
\hline $2016-2^{b}$ & 0.049 & 0.045 & 0.073 & 0.066 & 0.123 & 0.108 & 0.131 & 0.115 & 0.142 & 0.124 & 0.202 & 0.168 & 0.279 & 0.113 \\
\hline $2017-2^{b}$ & 0.101 & 0.069 & 0.138 & 0.103 & 0.167 & 0.145 & 0.165 & 0.143 & 0.162 & 0.139 & 0.148 & 0.122 & 0.118 & 0.092 \\
\hline $2013-3^{c}$ & 0.433 & 0.091 & 0.177 & 0.154 & 0.093 & 0.083 & 0.089 & 0.079 & 0.085 & 0.076 & 0.071 & 0.064 & 0.052 & 0.048 \\
\hline $2015-3^{c}$ & 0.047 & 0.043 & 0.070 & 0.063 & 0.118 & 0.104 & 0.126 & 0.110 & 0.136 & 0.119 & 0.198 & 0.168 & 0.305 & 0.112 \\
\hline $2016-3^{c}$ & 0.071 & 0.058 & 0.103 & 0.085 & 0.158 & 0.137 & 0.164 & 0.143 & 0.170 & 0.147 & 0.177 & 0.140 & 0.158 & 0.104 \\
\hline $2017-3^{c}$ & 0.077 & 0.060 & 0.110 & 0.088 & 0.162 & 0.141 & 0.167 & 0.145 & 0.170 & 0.147 & 0.169 & 0.135 & 0.145 & 0.101 \\
\hline
\end{tabular}

154 aThe model data for Figure $2 d$.

${ }^{\mathrm{b}}$ The model data for Figure 3c.

${ }^{c}$ The model data for Figure 3d. 


\section{Supporting Figures}
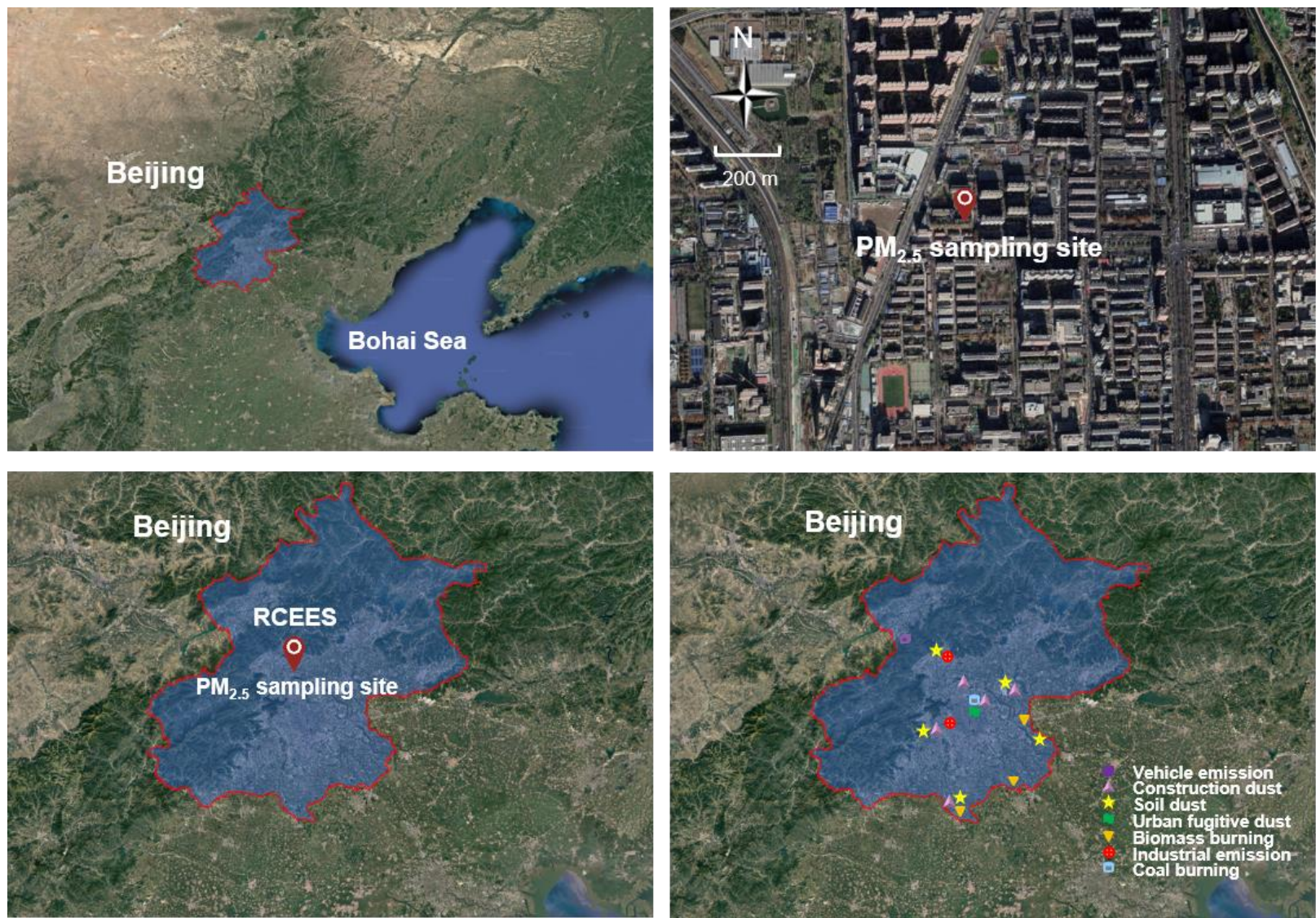

Figure S1. Sampling site. $\mathrm{PM}_{2.5}$ samples were collected in Beijing-a megacity that has experienced severe haze pollution during the past decades. Specifically, all $\mathrm{PM}_{2.5}$ samples were collected on a rooftop (about $25 \mathrm{~m}$ above ground) in the Research Center for Eco-Environment Sciences (RCEES, $40^{\circ} 00^{\prime} 29.85^{\prime \prime} \mathrm{N}, 116^{\circ} 20^{\prime} 29.71^{\prime \prime} \mathrm{E}$ ). There are two roads (one $150 \mathrm{~m}$ to the west and another $100 \mathrm{~m}$ to the south), universities, and residential areas nearby. The lower right picture shows the sampling sites for primary sources. The satellite images are obtained from the QGIS software (version 3.12.2). 


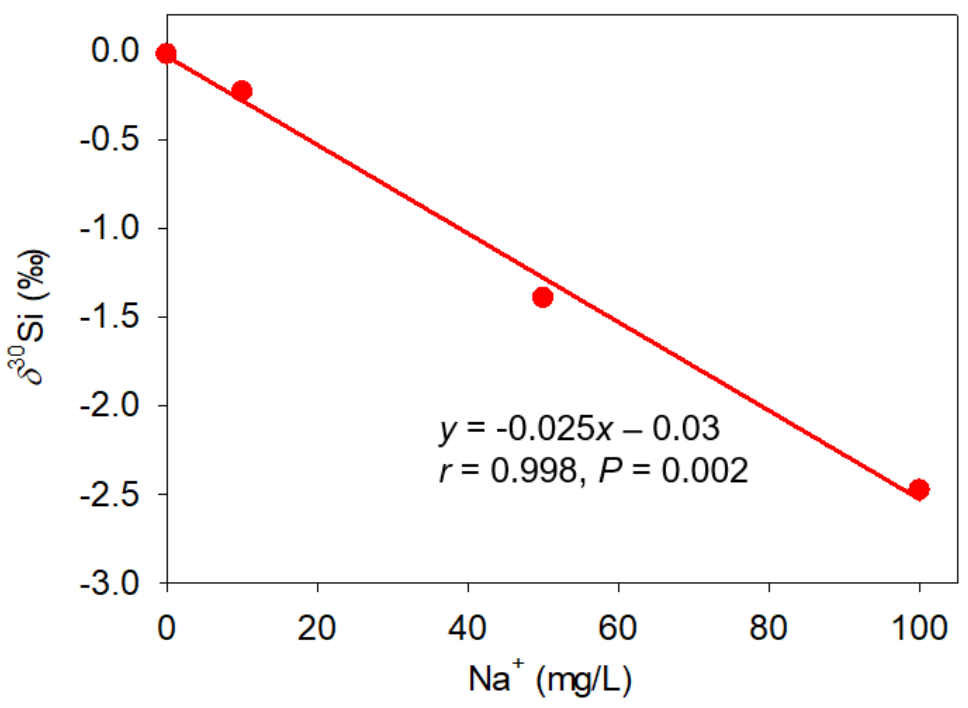

167 168 169 170 171

172 


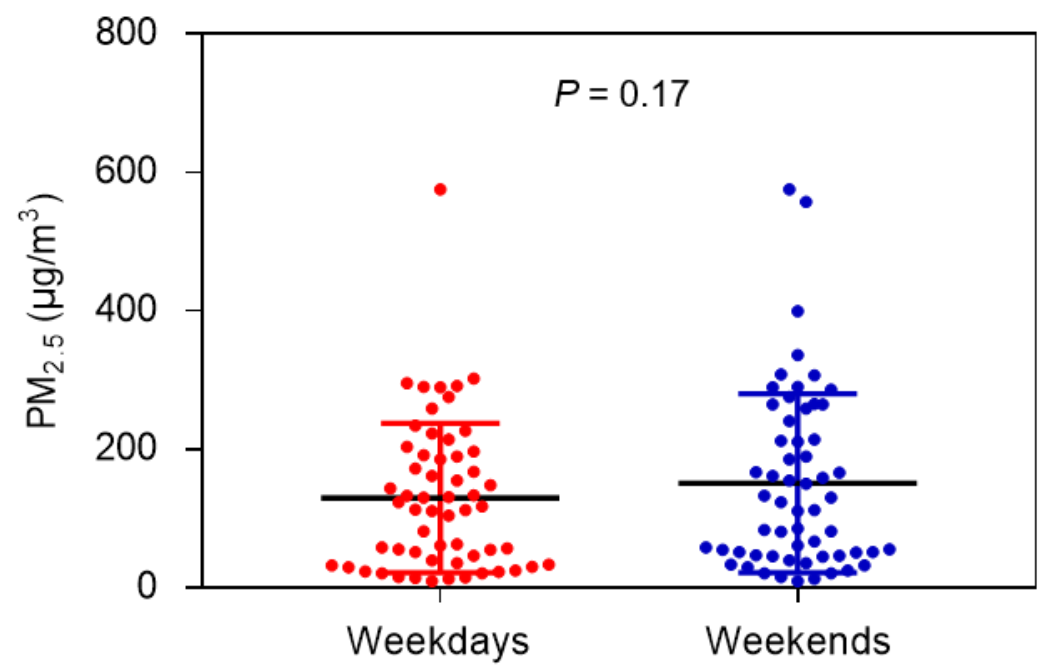

173

174 Figure S3. Pollution levels between weekdays and weekends. All PM2.5 samples were divided into 175 two groups, weekdays (from Monday to Friday) and weekends (Saturday and Sunday). Results showed 176 that no significant difference $(P=0.17)$ in $\mathrm{PM}_{2.5}$ concentration was observed between weekdays and 177 weekends.

178 

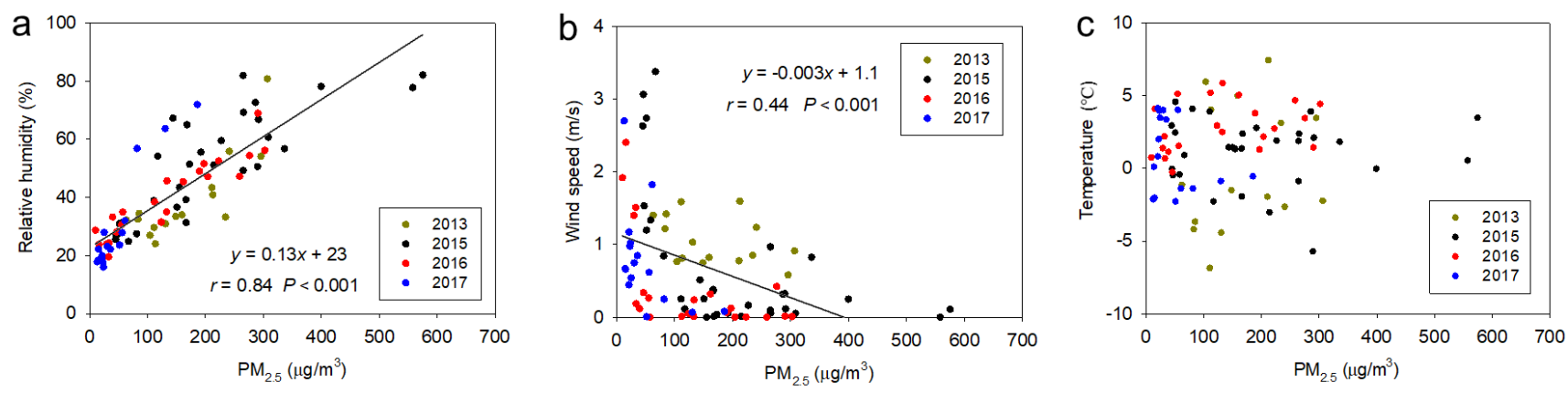

180 Figure S4. Correlation analyses of relative humidity (a), wind speed (b) and temperature (c) with $181 \mathbf{P M}_{2.5}$ concentration. The detailed data are shown in Table S1. 

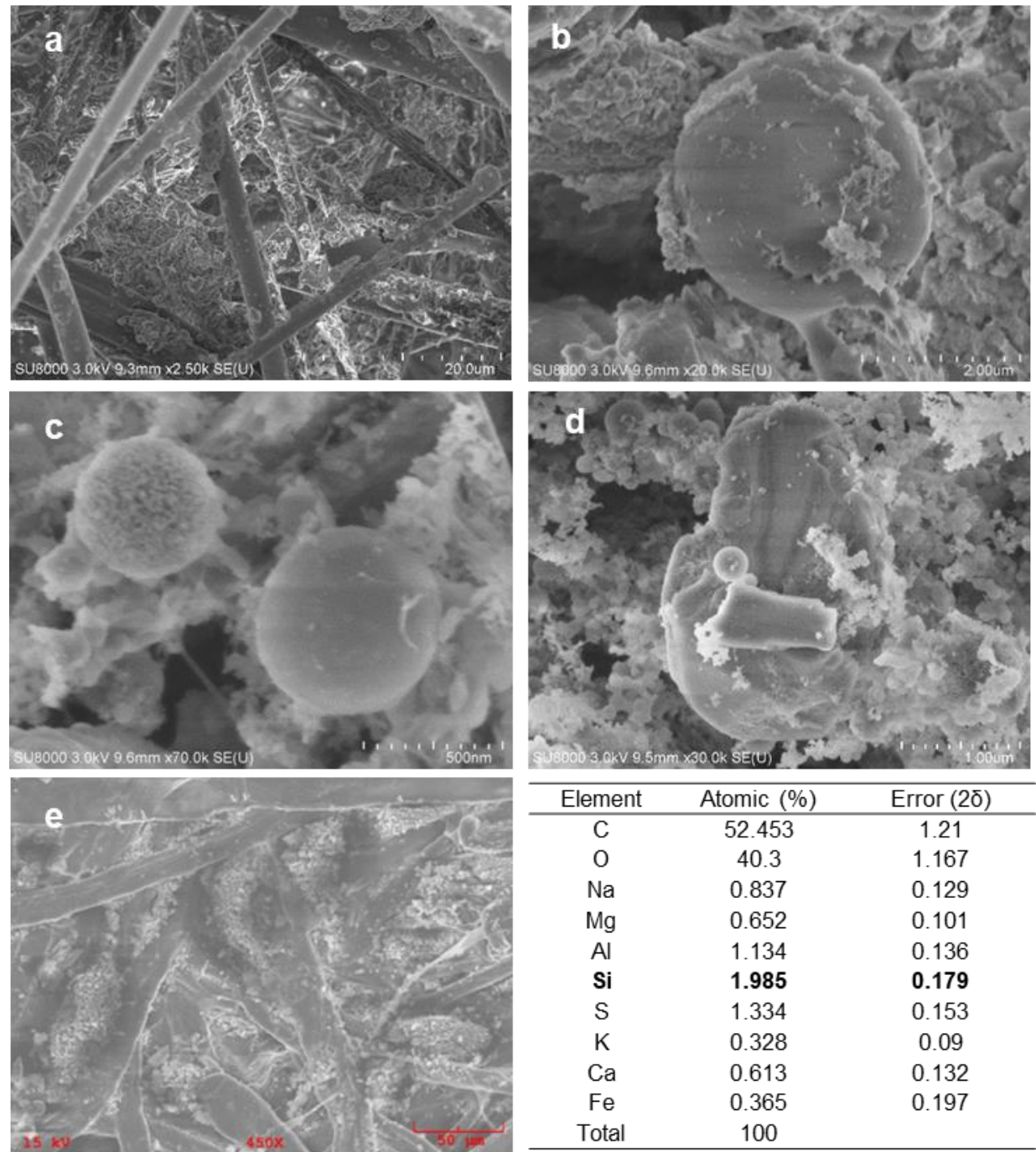

\begin{tabular}{ccc}
\hline Element & Atomic (\%) & Error (20) \\
\hline $\mathrm{C}$ & 52.453 & 1.21 \\
$\mathrm{O}$ & 40.3 & 1.167 \\
$\mathrm{Na}$ & 0.837 & 0.129 \\
$\mathrm{Mg}$ & 0.652 & 0.101 \\
$\mathrm{Al}$ & 1.134 & 0.136 \\
$\mathrm{Si}$ & $\mathbf{1 . 9 8 5}$ & $\mathbf{0 . 1 7 9}$ \\
$\mathrm{S}$ & 1.334 & 0.153 \\
$\mathrm{~K}$ & 0.328 & 0.09 \\
$\mathrm{Ca}$ & 0.613 & 0.132 \\
$\mathrm{Fe}$ & 0.365 & 0.197 \\
Total & 100 & \\
\hline
\end{tabular}

Figure S5. SEM and EDX analyses of several typical PM2.5 samples. a-c, SEM images of PM2.5 collected in light haze days $\left(60 \mu \mathrm{g} / \mathrm{m}^{3}\right.$ on 2016.01 .03 (a) and $35.3 \mu \mathrm{g} / \mathrm{m}^{3}$ on 2015.11.17 (b,c)). For (a), the $\mathrm{PM}_{2.5}$ sample was directly characterized on polypropylene sampling membranes. For (b) and (c), the $\mathrm{PM}_{2.5}$ samples were dissolved in ultrapure water and then dried in electroconductive pastes. The dried samples can be directly characterized by SEM. d, SEM image of PM2.5 collected in a heavy haze day $\left(274.0 \mu \mathrm{g} / \mathrm{m}^{3}, 2015.11 .14\right)$. This sample was prepared as that in (b) and (c). e, SEM image of PM 2.5 collected in a heavy haze day $\left(274.0 \mu \mathrm{g} / \mathrm{m}^{3}, 2015.11 .14\right)$ and the elemental composition given by EDX analysis. The EDX result confirmed the abundant presence of Si in $\mathrm{PM}_{2.5}$ as previously reported. 5, 6, 26 

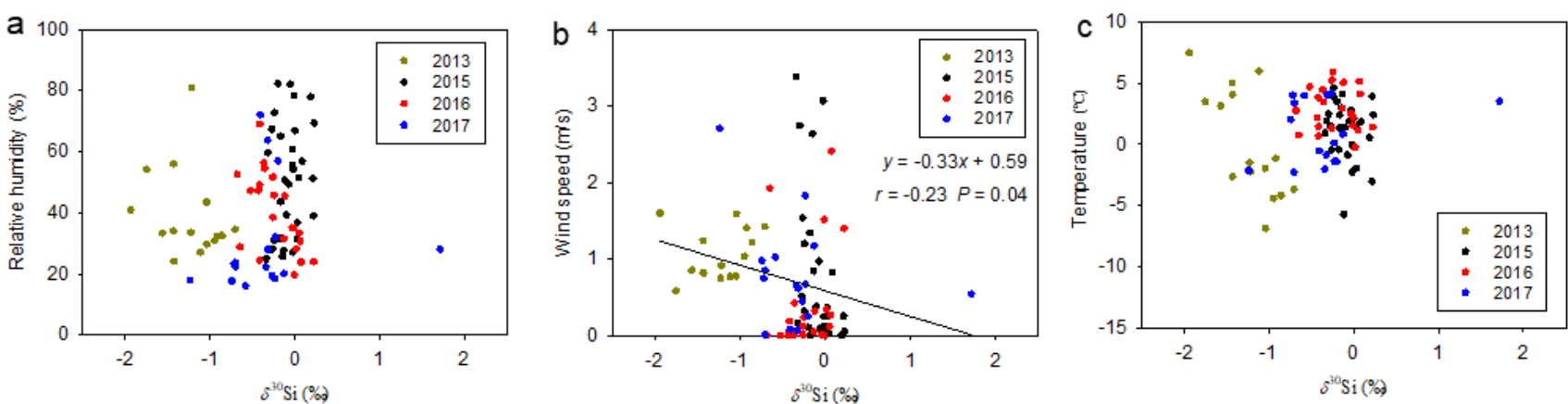

194 Figure S6. Correlation analyses of relative humidity (a), wind speed (b) and temperature (c) with 195 Si isotopic composition of $\mathbf{P M}_{2.5}$. The detailed data are shown in Table S5. 


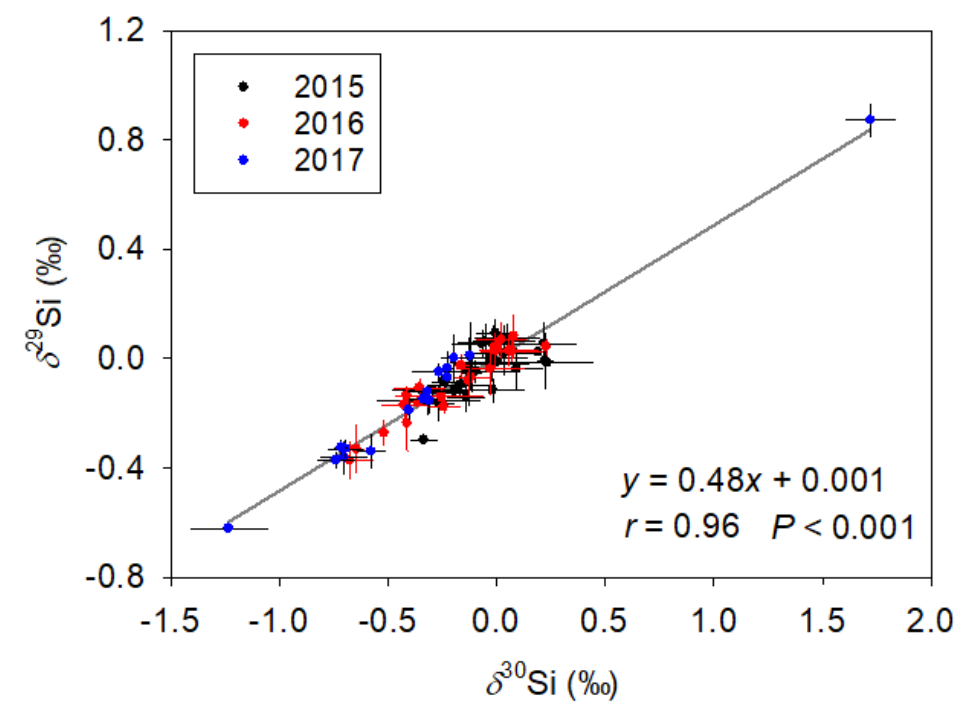

197

198 Figure S7. Three-isotope plots of $\delta^{\mathbf{2 9}}$ Si versus $\delta^{\mathbf{3 0}}$ Si for PM2.5 samples collected in Beijing during

199 2015-2017. It can be seen that most of samples fell onto the terrestrial mass-dependent fractionation

200 (MDF) line (within 1SD). A few samples (e.g., sample on 2015.12.07) showed derivations larger than

201 1SD from the MDF line. Here, the method uncertainty was 0.10\% (1SD) with standard reference 202 materials, and the SDs for $\delta^{29} \mathrm{Si}$ and $\delta^{30} \mathrm{Si}$ in real $\mathrm{PM}_{2.5}$ sample analysis were in the ranges of $0.01-$ $203 \quad 0.16 \%$ and $0.01-0.24 \%$ (see Table S1). Therefore, the PM2.5 samples seemed not to display significant 204 evidence of mass-independent fractionation (MIF). Similar phenomena have also been observed 205 previously with $\mathrm{Si}$ isotopes. ${ }^{27}$ 

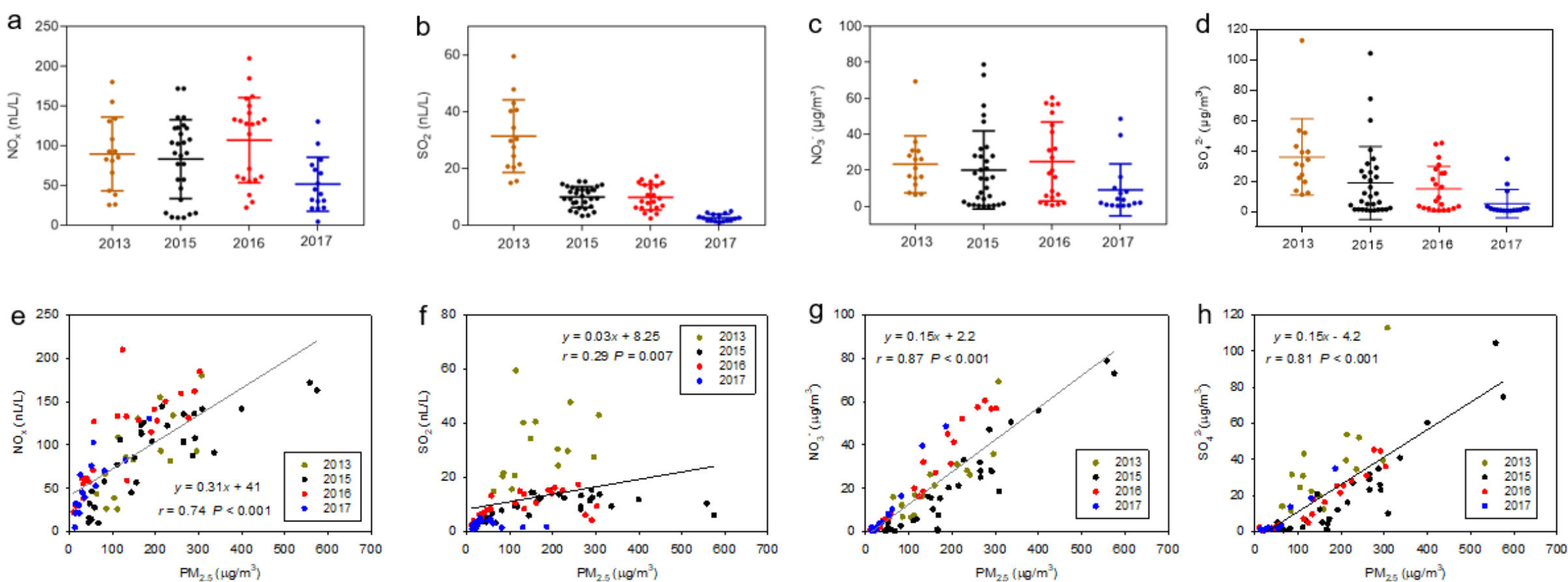

Figure S8. Annual ranges of secondary aerosol precursors $\left(\mathrm{NO}_{x}\right.$ and $\left.\mathrm{SO}_{2}\right)$ and secondary species $\left(\mathrm{NO}_{3}{ }^{-}\right.$and $\left.\mathrm{SO}_{4}{ }^{2-}\right)$ and their correlation analyses with the $\mathbf{P M}_{2.5}$ concentration. a-d, Annual ranges of $\mathrm{NO}_{x}(\mathbf{a}), \mathrm{SO}_{2}$ (b), $\mathrm{NO}_{3}{ }^{-}$(c) and $\mathrm{SO}_{4}{ }^{2-}$ (d) from 2013 to 2017 . e-h, Correlation analyses of $\mathrm{NO}_{x}(\mathbf{e}), \mathrm{SO}_{2}(\mathbf{f}), \mathrm{NO}_{3}{ }^{-}(\mathbf{g})$ and $\mathrm{SO}_{4}{ }^{2-}(\mathbf{h})$ with the $\mathrm{PM}_{2.5}$ concentration. The detailed data are shown in $\mathbf{T a b l e} \mathbf{S 1}$ and $\mathbf{S 5}$. $\mathrm{SO}_{2}$ is thought to be mainly emitted from coal burning and industrial emission. From $\mathbf{b}$ and $\mathbf{d}, \mathrm{SO}_{2}$ and $\mathrm{SO}_{4}{ }^{2-}$ showed a sharp decline during $2013-2015$ and then changed slowly during 2015-2017. This trend is consistent with the variation trends of coal burning and industrial emission in Figure 2d. $\mathrm{NO}_{x}$ and $\mathrm{NO}_{3}{ }^{-}$, with more complex sources, showed a much slower decreasing trend, which was also consistent with that in previous reports. ${ }^{19}$ 


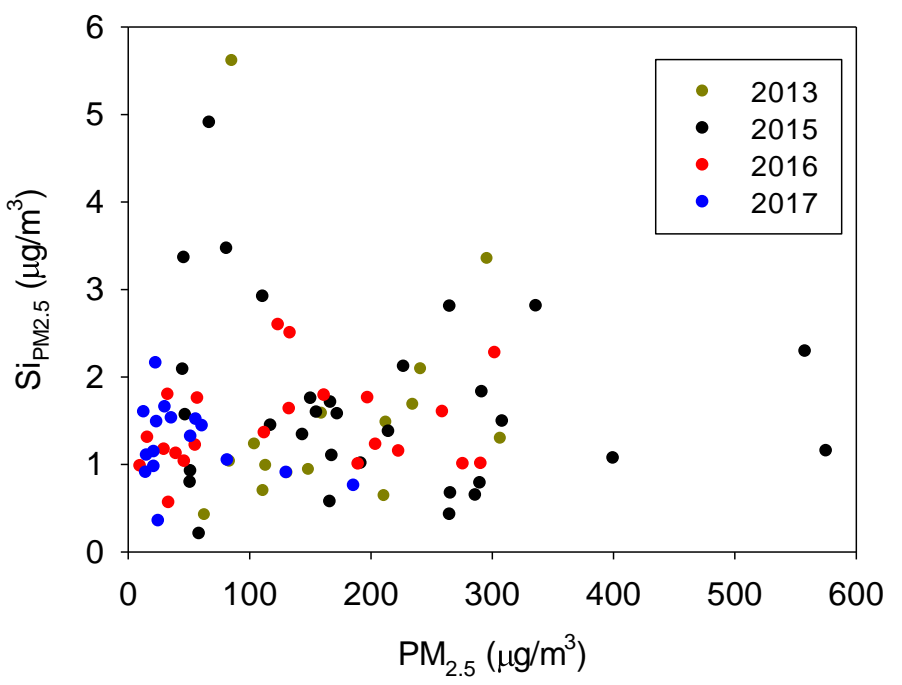

215

216 Figure S9. Correlations analysis of Si concentration with $\mathbf{P M}_{2.5}$ concentration during 2013-2017.

217 The Si concentration exhibited no correlation with the pollution level during 2013-2017. Considering 218 the high secondary aerosol contributions in these years (all > 42\%), with the pollution level increasing, 219 the amounts of secondary aerosols would increase. But the Si concentration did not show a 220 synchronous increasing trend, which was consistent with the hypothesis that the total Si in PM 2.5 was 221 not affected by secondary aerosol formation process. 

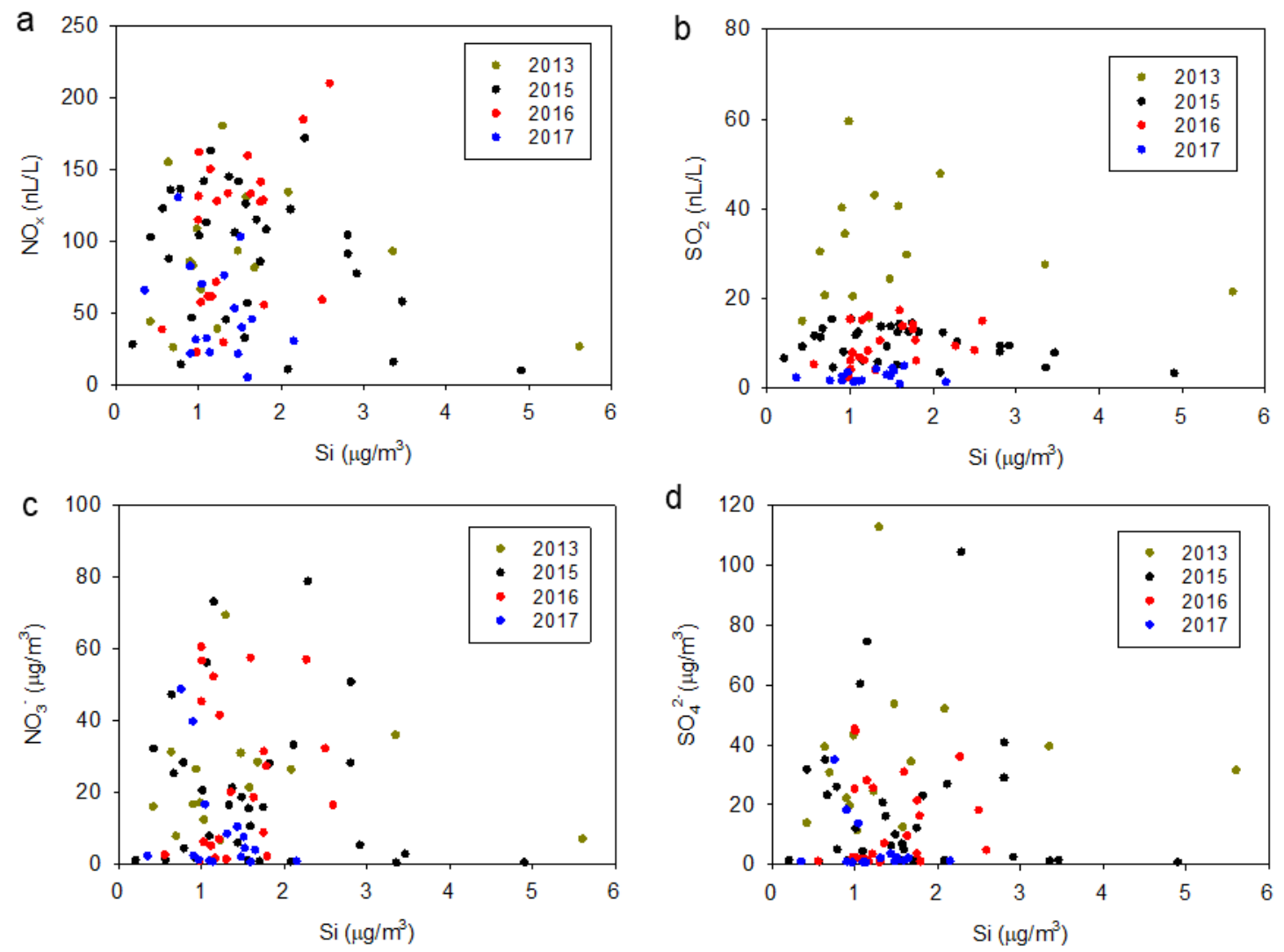

224 Figure S10. Correlation analyses of $\mathrm{NO}_{\mathrm{x}}(\mathrm{a}), \mathrm{SO}_{2}(\mathrm{~b}), \mathrm{NO}_{3}^{-}(\mathrm{c})$, and $\mathrm{SO}_{4}^{2-}$ (d) with the $\mathrm{Si}$ 225 concentration in $\mathbf{P M}_{2.5}$ during 2013-2017. The detailed data are shown in Table S5. The Si concentration showed no correlations with $\mathrm{NO}_{x}, \mathrm{SO}_{2}, \mathrm{NO}_{3}^{-}$, and $\mathrm{SO}_{4}{ }^{2-}$, also suggesting that the $\mathrm{Si}$ content in $\mathrm{PM}_{2.5}$ was not affected by the secondary formation process. 


\section{References for SI}

230 1. Huang, R. J.; Zhang, Y.; Bozzetti, C.; Ho, K. F.; Cao, J. J.; Han, Y.; Daellenbach, K. R.; Slowik, J. 231 G.; Platt, S. M.; Canonaco, F.; Zotter, P.; Wolf, R.; Pieber, S. M.; Bruns, E. A.; Crippa, M.; Ciarelli, G.; 232 Piazzalunga, A.; Schwikowski, M.; Abbaszade, G.; Schnelle-Kreis, J.; Zimmermann, R.; An, Z.; Szidat, S.; Baltensperger, U.; El Haddad, I.; Prevot, A. S. High secondary aerosol contribution to particulate pollution during haze events in China. Nature 2014, 514 (7521), 218-222.

2. Zhang, R.; Jing, J.; Tao, J.; Hsu, S.-C.; Wang, G.; Cao, J.; Lee, C. S. L.; Zhu, L.; Chen, Z.; Zhao, Y. Chemical characterization and source apportionment of $\mathrm{PM}_{2.5}$ in Beijing: Seasonal perspective. Atmos. Chem. Phys. 2013, 13 (14), 7053-7074.

3. Song, Y.; Tang, X.; Xie, S.; Zhang, Y.; Wei, Y.; Zhang, M.; Zeng, L.; Lu, S. Source apportionment of $\mathrm{PM}_{2.5}$ in Beijing in 2004. J. Hazard. Mater. 2007, 146 (1), 124-130.

4. He, K.; Yang, F.; Ma, Y.; Zhang, Q.; Yao, X.; Chan, C. K.; Cadle, S.; Chan, T.; Mulawa, P. The characteristics of $\mathrm{PM}_{2.5}$ in Beijing, China. Atmos. Environ. 2001, 35 (29), 4959-4970.

5. Lu, D.; Tan, J.; Yang, X.; Sun, X.; Liu, Q.; Jiang, G., Unraveling the role of silicon in atmospheric aerosol secondary formation: a new conservative tracer for aerosol chemistry. Atmos. Chem. Phys. 2019, 19 (5), 2861-2870.

6. Lu, D.; Liu, Q.; Yu, M.; Yang, X.; Fu, Q.; Zhang, X.; Mu, Y.; Jiang, G., Natural silicon isotopic signatures reveal the sources of airborne fine particulate matter. Environ. Sci. Technol. 2018, 52 (3), 1088-1095.

7. Ge, S.; Xu, X.; Chow, J. C.; Watson, J.; Sheng, Q.; Liu, W.; Bai, Z.; Zhu, T.; Zhang, J. Emissions of air pollutants from household stoves: honeycomb coal versus coal cake. Environ. Sci. Technol. 2004, 38 (17), 4612-4618.

8. Fraser, M. P.; Cass, G. R.; Simoneit, B. R. Gas-phase and particle-phase organic compounds emitted from motor vehicle traffic in a Los Angeles roadway tunnel. Environ. Sci. Technol. 1998, 32 (14), 2051-2060.

9. Liu, P.; Zhang, C.; Mu, Y.; Liu, C.; Xue, C.; Ye, C.; Liu, J.; Zhang, Y.; Zhang, H., The possible contribution of the periodic emissions from farmers' activities in the North China Plain to atmospheric water-soluble ions in Beijing. Atmos. Chem. Phys. 2016, 16 (15), 10097.

10. Houlton, B. Z.; Sigman, D. M.; Schuur, E. A.; Hedin, L. O., A climate-driven switch in plant nitrogen acquisition within tropical forest communities. Proc. Natl. Acad. Sci. U. S. A. 2007, 104 (21), 8902-8906.

11. Pan, Y.; Tian, S.; Liu, D.; Fang, Y.; Zhu, X.; Zhang, Q.; Zheng, B.; Michalski, G.; Wang, Y., Fossil fuel combustion-related emissions dominate atmospheric ammonia sources during severe haze 
episodes: Evidence from ${ }^{15} \mathrm{~N}$-stable isotope in size-resolved aerosol ammonium. Environ. Sci. Technol. 2016, 50 (15), 8049-8056.

12. Yu, X.; Zhang, J.; Kong, F.; Li, Y.; Li, M.; Dong, Y.; Xi, M., Identification of source apportionment and its spatial variability of dissolved organic matter in Dagu River-Jiaozhou Bay estuary based on the isotope and fluorescence spectroscopy analysis. Ecol. Indict. 2019, 102, 528-537.

13. Guo, S.; Hu, M.; Guo, Q.; Zhang, X.; Zheng, M.; Zheng, J.; Chang, C. C.; Schauer, J. J.; Zhang, R., Primary sources and secondary formation of organic aerosols in Beijing, China. Environ. Sci. Technol. 2012, 46 (18), 9846-9853.

14. Zhou, J.; Xiong, Y.; Xing, Z.; Deng, J.; Du, K., Characterizing and sourcing ambient $\mathrm{PM}_{2.5}$ over key emission regions in China II: Organic molecular markers and CMB modeling. Atmos. Environ. 2017, 163, 57-64.

15. Kea, L.; Liu, W.; Wang, Y.; Russellc, A. G.; Edgertond, E. S.; Zheng, M., Comparison of PM2.5 source apportionment using positive matrix factorization and molecular marker-based chemical mass balance. Sci. Total Environ. 2008, 394 (2-3), 290-302.

16. Schmid, H.; Laskus, L.; Abraham, H. J.; Baltensperger, U.; Lavanchy, V.; Bizjak, M.; Burba, P.; Cachier, H.; Crow, D.; Chow, J.; Gnauk, T.; Even, A.; ten Brink, H. M.; Giesen, K. P.; Hitzenberger, R.; Hueglin, C.; Maenhaut, W.; Pio, C.; Carvalho, A.; Putaud, J. P.; Toom-Sauntry, D.; Puxbaum, H., Results of the "carbon conference" international aerosol carbon round robin test stage I, Atmos. Environ. 2001, 35, 2111-2121.

17. Marcazzan, G. M.; Ceriani, M.; Valli, G.; Vecchi, R., Source apportionment of $\mathrm{PM}_{10}$ and $\mathrm{PM}_{2.5}$ in Milan (Italy) using receptor modelling. Sci. Total Environ. 2003, 317 (1-3), 137-147.

18. Cheng, J.; Su, J.; Cui, T.; Li, X.; Dong, X.; Sun, F.; Yang, Y.; Tong, D.; Zheng, Y.; Li, Y.; Li, J.; Zhang, Q.; He, K., Dominant role of emission reduction in $\mathrm{PM}_{2.5}$ air quality improvement in Beijing during 2013-2017: A model-based decomposition analysis. Atmos. Chem. Phys. 2019, 19 (9), 61256146.

19. Li, H.; Cheng, J.; Zhang, Q.; Zheng, B.; Zhang, Y.; Zheng, G.; He, K., Rapid transition in winter aerosol composition in Beijing from 2014 to 2017: Response to clean air actions. Atmos. Chem. Phys. 2019, 19 (17), 11485-11499.

20. Yang, X.; Liu, X.; Zhang, A.; Lu, D.; Li, G.; Zhang, Q.; Liu, Q.; Jiang, G., Distinguishing the sources of silica nanoparticles by dual isotopic fingerprinting and machine learning. Nat. Commun. 2019, 10, 1620-1628.

21. Geilert, S.; Vroon, P. Z.; van Bergen, M. J., Effect of diagenetic phase transformation on the silicon isotope composition of opaline sinter deposits of Geysir, Iceland. Chem. Geol. 2016, 433, 57-67.

22. Geilert, S.; Vroon, P. Z.; Keller, P. S.; Gudbrandsson, S.; Stefansson, A.; van Bergen, M. J.; Silicon 
296 isotope fractionation during silica precipitation from hot-spring waters: Evidence from the Geysir 297 geothermal field, Iceland. Geochim. Cosmochim. Acta 2015, 164, 403-427.

298 23. Emma Engström, E.; Rodushkin, I.; Baxter, D. aca.; Öhlander, B., Chromatographic purification 299 for the determination of dissolved silicon isotopic compositions in natural waters by high-resolution 300 multicollector inductively coupled plasma mass spectrometry. Anal. Chem. 2006, 78, 250-257.

301 24. Andrade, C. N.; Chafetz, H. S.; Lapen, T. J., Paragenesis of silicified mid-Paleozoic and mid302 Cenozoic corals based on petrography and silicon isotopic analyses. Chem. Geol. 2020, 538, 119483. 303 25. Li, M.; Zhang, Q.; Kurokawa, J.-i.; Woo, J.-H.; He, K.; Lu, Z.; Ohara, T.; Song, Y.; Streets, D. G.; 304 Carmichael, G. R.; Cheng, Y.; Hong, C.; Huo, H.; Jiang, X.; Kang, S.; Liu, F.; Su, H.; Zheng, B., MIX: 305 a mosaic Asian anthropogenic emission inventory under the international collaboration framework of 306 the MICS-Asia and HTAP. Atmos. Chem. Phys. 2017, 17 (2), 935-963.

307 26. Bzdek, B. R.; Horan, A. J.; Pennington, M. R.; Janechek, N. J.; Baek, J.; Stanier, C. O.; Johnston, 308 M. V., Silicon is a frequent component of atmospheric nanoparticles. Environ. Sci. Technol. 2014, 48 309 (19), 11137-11145.

310 27. Zambardi, T.; Poitrasson, F., Precise determination of silicon isotopes in silicate rock reference 311 materials by MC-ICP-MS. Geostand. Geoanal. Res. 2011, 35 (1), 89-99. 